\title{
Making Contact with Observations
}

\section{Ioannis Votsis}

\author{
University of Duesseldorf
}

\section{Introduction}

Prajit K. Basu (2003) argues that for observations to be of use in theory testing, they first need to be transformed into evidence. Since the transformation, according to him, involves the introduction of theoretical vocabulary, the end-product is theory-laden. ${ }^{1}$ Basu motivates his claims using a distinction between raw (observational) data and evidence, adapted from Bogen and Woodward's influential distinction between data and phenomena. ${ }^{2}$ Following Bogen and Woodward, he claims that theories do not entail, predict or explain observation statements or data, not even with the help of suitable theoretical auxiliaries. This prevents any direct observational assessment of theories (plus auxiliaries). ${ }^{3}$ My aim in this paper is to contest the claim that theories accompanied by suitable theoretical auxiliaries cannot be

\footnotetext{
${ }^{1}$ Basu states: "A piece of evidence is then expressed by a sentence in the language, LT, of the theory. It is in this sense that a piece of evidence is theory laden" (p. 358).

${ }^{2}$ According to Bogen and Woodward, data are observable whereas (physical) phenomena are unobservable. Theories only talk about the latter. As they stress, “...data typically cannot be predicted or systematically explained by theory" (1988, pp. 305-306). Although Basu agrees with much of what Bogen and Woodward have to say, he thinks that their distinction "is inadequate in handling cases of 'revolutions' in science" (p. 354).

${ }^{3}$ Observations, Basu claims, need not be theory-laden but they cannot play a direct role in confirmation: "...although one could legitimately hold that there are observations that are not theory infected, such observations cannot be employed for theory resolution” (2003, p. 356) [my emphasis]. Confusingly, his conception of 'non-theory laden' observations is anything but 'non-theory laden', as he takes them to be those that scientists can merely agree on. He says: "Observations understood as (raw) data are trivially theory-laden. But in a more interesting sense, they may not be. This is because the consensus within the scientific community about what observations (or raw data) are, does not emphasize the ineliminably theoretical aspect these observations have. This consensus is historically contingent” (p. 364).
} 
directly tested via observations. In so doing, I will utilise Basu's own case study, a rather well-known controversy between Antoine Lavoisier and Joseph Priestly.

The Lavoisier-Priestly controversy concerns two conflicting results emanating from what appear to be the same experiments independently carried out by the two scientists. Both scientists were in agreement that the observable result of the experiments was the production of a black powder with certain properties. ${ }^{4}$ Since their respective theories of oxygen and of phlogiston do not speak of (or indeed entail) the presence of black powder, the observable result cannot immediately be used for theory adjudication. The raw observational data first has to be theoretically treated. This is where the disagreement arose. For Priestly, who advocated the phlogiston theory, when iron is heated in dephlogisticated air it leads to the production of iron calx. For Lavoisier, an advocate of the oxygen theory, the heating of iron in oxygen leads to the production of iron oxide. Yet, the presence of iron calx is only entailed by the phlogiston theory and the presence of iron oxide is only entailed by the oxygen theory. In other words, the same observation (i.e. the presence of a particular kind of black powder) is theoretically interpreted - out of necessity, for on its own, Basu claims, it is not evidentially potent - as two different evidential statements, each only confirming its respective theory.

Although Basu takes theoretical auxiliaries as necessary for the transformation of observations into evidence, he insists that they cannot help infer the relevant observation statements from the given theory. In the case at hand, this means that the presence of that particular kind of black powder cannot be inferred from either of the two theories. To see this

\footnotetext{
${ }^{4}$ Priestly and Lavoisier agreed on various other observable results such as balance readings. They disagreed on whether the reaction only led to the production of black powder, namely Priestly thought that carbon dioxide was also produced. This disagreement is not important for our current discussion - Basu similarly sidelines it - as we are only interested in the inferences that take us from (commonly shared) observations to evidence.
} 
point, let's formalise the aforementioned statements. Let $\mathrm{O}_{1}$ : Iron is heated in oxygen, $\mathrm{O}_{2}$ : Iron is heated in dephlogisticated air, $\mathrm{E}_{1}$ : Iron oxide is produced, $\mathrm{E}_{2}$ : Iron calx is produced, $\mathrm{B}$ : Black powder with certain observable properties is present, $\mathrm{L}: \mathrm{O}_{1} \rightarrow \mathrm{E}_{1}, \mathrm{P}: \mathrm{O}_{2} \rightarrow \mathrm{E}_{2}, \mathrm{~A}_{1}$ : $\mathrm{B} \rightarrow$ $\mathrm{E}_{1}$ and $\mathrm{A}_{2}: \mathrm{B} \rightarrow \mathrm{E}_{2}$. The relevant statement in Lavoisier's theory is L and the one in Priestly's theory is $\mathrm{P} . \mathrm{A}_{1}$ and $\mathrm{A}_{2}$ are theoretical auxiliaries that respectively allow each scientist to go from observation to evidence. ${ }^{5}$ Let's take Lavoisier's theory first. From $\mathrm{O}_{1}$ and $\mathrm{L}$, we can infer $\mathrm{E}_{1}$ but not $\mathrm{B}$. To confirm Lavoisier's theory we must assume $\mathrm{A}_{1}$ which together with $\mathrm{B}$ entail $\mathrm{E}_{1}$. Thus, to confirm Lavoisier's theory (or at least one of its parts, i.e. L), we must first transform $\mathrm{B}$ into an evidentially relevant statement (i.e. $\mathrm{E}_{1}$ ) using theoretical auxiliary $\mathrm{A}_{1}$. Notice that if we add $A_{1}$ to the set of statements $\left\{\mathrm{O}_{1}, L\right\}$ we still cannot infer $B$. This seems to vindicate Basu's point that even with the help of theoretical auxiliaries we cannot infer the observational statement. In his own words, “...the construction of $E_{1}$ in (1) [i.e. the proposition that $\mathrm{B}$ and $\mathrm{A}_{1}$ imply $E_{1}$ ] is asymmetrical. The fact that iron oxide is produced does not entail (along with $\left[\mathrm{A}_{1}\right]$ ) that a black powder is produced" (p. 361). ${ }^{6}$ The same can be said of Priestly's theory. From $\mathrm{O}_{2}$ and $\mathrm{P}$, we can infer $\mathrm{E}_{2}$ but not B. To confirm Priestly's theory we must assume $\mathrm{A}_{2}$ which together with $\mathrm{B}$ entail $\mathrm{E}_{2}$. Thus, to confirm Priestly's theory (or at least one of its parts, i.e. P), we must first transform B into an evidentially relevant statement (i.e. $\mathrm{E}_{2}$ ) using theoretical auxiliary $\mathrm{A}_{2}$. Again, notice that if we add $\mathrm{A}_{2}$ to the set of statements $\left\{\mathrm{O}_{2}, \mathrm{P}\right\}$ we cannot infer B. Finally, it is worth mentioning that we cannot judge Priestly's theory on $\mathrm{E}_{1}$ and Lavoisier's theory on $\mathrm{E}_{2}$. Each evidential statement is at best irrelevant to the other theory, at worst disconfirms it.

\footnotetext{
${ }^{5}$ The auxiliaries are conjunctions of various theses. Both $\mathrm{A}_{1}$ and $\mathrm{A}_{2}$ include the Stahlian theses, as Basu suggests (p. 361).

${ }^{6}$ Basu uses different letters to denote the auxiliaries.
} 
Basu ponders at one point "whether it is possible to predict the (raw) data from the hypothesis by employing suitable auxiliary assumptions" (p. 362). He effectively dismisses this possibility. In a footnote he says: "In fact, there are indefinite number of such assumptions that need to be specified... that seems to be a hard, if not impossible task" (p. 363, 35f) [my emphasis]. Indeed, he thinks that "(raw) data never have any evidential bearing" (p. 364) [my emphasis]. In what follows, I contest the claim that it is hard to find suitable auxiliary assumptions that let us derive, predict and potentially explain observational reports like B.

\section{Partitioning Properties}

Sets can be partitioned into various disjoint parts. More formally we say that a set $\mathrm{P}$ is a partition of a set $S$ if and only if (1) all of P's members are non-empty subsets of $S$, (2) the union of P's members is co-extensional to $\mathrm{S}$ and (3) the intersection of any two members of $\mathrm{P}$ is empty. ${ }^{7}$ A peculiar aspect of this standard definition is that any set $\mathrm{S}$ (that can be partitioned) will have $\{\mathrm{S}\}$ among its partitions. ${ }^{8}$ For those interested in splitting the original set into two or more disjoint parts, a partition containing the original set as a member will of course be unwanted. To overcome this problem, let's define another notion that prohibits such partitions, call it 'partition*'. A set $\mathrm{P}$ is a partition* of a set $\mathrm{S}$ if and only if $\mathrm{P}$ fulfils the above three conditions (i.e. it is a partition of $\mathrm{S}$ ) and $\mathrm{P}$ does not contain $\mathrm{S}$ as a member. ${ }^{9}$ Let's denote

\footnotetext{
${ }^{7}$ An alternative first condition does not exclude non-empty subsets of S, thereby allowing for partitions such as $\{\mathrm{S}, \varnothing\}$.

${ }^{8}$ This means that even a singleton set has a partition - in fact it has only one partition. For example set $\{1\}$ has one and only one partition, namely $\{\{1\}\}$.

${ }^{9}$ Alternatively we may modify the first condition so that it reads 'all of P's members are non-empty proper subsets of S'.
} 
such a set as $\operatorname{Part}^{*}(\mathrm{~S})$. Sets with less than two members cannot be partitioned*. For a set $\mathrm{S}$ with $n$ members, the number of partitions* is given by the bell number of that set minus one. ${ }^{10}$

Let us now move from propositional to predicate logic. ${ }^{11}$ Predicates denote properties. Extensionally understood, properties are sets. That means that for any set there is one and only corresponding (natural or artificial) property, and vice-versa. This allows us to partition* properties by partitioning* their corresponding sets. Thus a partition* of a set $\mathrm{S}$ will have as members non-empty non-intersecting sets, each of which can be assigned a different property. Indeed, any property applicable to more than one object can be partitioned ${ }^{*}$ into two or more properties each of which is distinct from one another and applicable to at least one object. Take the property of being a mammal. It can be partitioned* into a great number of properties, some of them corresponding to natural, others to artificial properties. Examples of (presumably) natural properties are the properties of primate, rodent, bat and dolphin. Examples of artificial properties are the properties of being half a meter long, being named 'Alexa' and weighing more than 500kg. ${ }^{12}$

To remove any lingering unclarity, let us take a closer look at an example of a set being partitioned*. Suppose $S=\{1,2,3\}$. We know that this set has four partitions*, i.e. $\operatorname{Part}_{1} *(S)=$

\footnotetext{
${ }^{10}$ The bell number of a set is the number of that set's partitions. For $n=1,2,3, \ldots$ the corresponding bell numbers are $1,2,5,15,52,203, \ldots$ Since partition* excludes $S$ itself as a legitimate 'partition', the number of partitions* of a set $\mathrm{S}$ is given by the bell number of that set minus one. That means for $n=1,2,3, \ldots$ the corresponding numbers of partitions* are $0,1,4,14,51,202, \ldots$

${ }^{11}$ The material presented in the previous section was presented in propositional form for expediency.

${ }^{12}$ Overlapping properties such as being half a meter long and being named 'Alexa' do not of course belong to the same partitions* of the property mammals. Note also that these particular properties are not contained whole in the property of being a mammal since there are non-mammals that instantiate them, i.e. strictly speaking they should be formulated as "being a mammal half a meter long" and "being a mammal named "Alexa' ".
} 
$\{\{1\},\{2\},\{3\}\}, \operatorname{Part}_{2} *(S)=\{\{1,2\},\{3\}\}, \operatorname{Part}_{3} *(S)=\{\{1,3\},\{2\}\}, \operatorname{Part}_{4} *(S)=\{\{2,3\}$, $\{1\}\}$. Observe that each partition* contains as members sets that are mutually disjoint and whose union is set S. Qua sets, each member of a partition* of S can be assigned a property. Take for example Part* ${ }_{1}(\mathrm{~S})$. It contains three members, namely sets $\{1\},\{2\},\{3\}$. Each of these can be assigned a different property; we can use the predicates $R_{1}, R_{2}$ and $R_{3}$ to denote these properties. Now if $\mathrm{R}$ is the predicate denoting the property corresponding to set $\mathrm{S}$, then ( $x)\left(R x \equiv\left(R_{1} x \vee R_{2} x \vee R_{3} x\right)\right)$. All the partitions* of $S$ can be given the same treatment. This is so, as the properties on the right side of the biconditional are jointly co-extensional to the property on the left side.

With these tools and results in mind, let us turn to the problem at hand. Given our move to predicate logic, atomic propositions $\mathrm{O}_{1}, \mathrm{O}_{2}, \mathrm{E}_{1}, \mathrm{E}_{2}$ and $\mathrm{B}$ are now taken to be predicates while complex propositions $\mathrm{L}, \mathrm{P}, \mathrm{A}_{1}$ and $\mathrm{A}_{2}$ are now quantified propositions. For example, theoretical auxiliary $\mathrm{A}_{1}$ now reads: $(\mathrm{x})\left(\mathrm{Bx} \rightarrow \mathrm{E}_{1} \mathrm{x}\right)$. Crucially, this universal generalisation implies that either $\mathrm{E}_{1}$ is co-extensional to $\mathrm{B}$ or $\mathrm{B}$ is a non-empty proper subset of $\mathrm{E}_{1} \cdot{ }^{13}$ In the former case, this amounts to the bi-conditional statement $\mathrm{A}_{3}:(\mathrm{x})\left(\mathrm{Bx} \equiv \mathrm{E}_{1} \mathrm{x}\right)$. If we add $\mathrm{A}_{3}$ as an auxiliary to our original set of propositions $\left\{\mathrm{O}_{1} a\right.$, L: $\left.(\mathrm{x})\left(\mathrm{O}_{1} \mathrm{x} \rightarrow \mathrm{E}_{1} \mathrm{x}\right)\right\}$ we can derive the desired sentence $\mathrm{B} a$, where $a$ is the particular object that bears these properties. In the latter case, we can turn to the concept of partition* to derive an equally suitable statement. We know that $\mathrm{B}$, qua a non-empty proper subset of $\mathrm{E}_{1}$, belongs to at least one partition* of $\mathrm{E}_{1} .^{14}$

\footnotetext{
${ }^{13}$ For simplicity, I use the same letters to denote predicates and their corresponding properties and sets. Context will determine which one I have in mind.

${ }^{14}$ Although some partitions* of $\mathrm{E}_{1}$ might not have $\mathrm{B}$ as a member, their members' union will contain all the objects that are contained in B. From these we can reconstruct B, e.g. by further partitioning* the members of a given partition* and then taking the relevant union of the resulting partitions*. That means that the partition*
} 
Take such a partition*, let's call it 'C'. C is co-extensional to $\mathrm{E}_{1}$. It contains $\mathrm{B}$ as a member but also one or more other sets that are disjoint from B. We can assign a property and hence a predicate to each of them. Let us call these ' $\mathrm{C}_{1}$ ',,${ }^{\prime} \mathrm{C}_{\mathrm{m}}$ ', where $m$ is determined by the number of disjoint sets in $\mathrm{C}$ other than $\mathrm{B}$. The following auxiliary can now be formulated $\mathrm{A}_{4}$ : (x) $\left(\mathrm{E}_{1} \mathrm{x} \equiv\left(\mathrm{Bx} \vee \mathrm{C}_{1} \mathrm{x} \vee \ldots \vee \mathrm{C}_{\mathrm{m}} \mathrm{x}\right)\right)$. The properties on the right side of the biconditional are jointly co-extensional to the property on the left side. If we add $\mathrm{A}_{4}$ to our original set of propositions we can derive the following conclusion $\mathrm{B} a \mathrm{v} \mathrm{C}_{1} a \ldots \mathrm{v}_{\mathrm{m}} a{ }^{15}$ The observational statement $\mathrm{B} a$ can confirm our conclusion without first being transformed into theory-laden evidence. ${ }^{16}$ Q.E.D.

I have just proved what Basu denied. Technicalities aside, the conclusion is supported by a very simple logical point. Suppose we are faced with the sort of presumed asymmetry Basu talks about, i.e. we have a statement of the form 'All F's are G's' but we really want a statement of the form 'All G's are F's' or at least some statement that allows us to go from G's to F's. If we know that all objects with property $F$ have property $G$, we can infer that either some objects with property $\mathrm{G}$ have property $\mathrm{F}$ or all of them do. The latter case plays straight into our hands. The former needs a little spelling out. That's where the partition* notion comes in, as it facilitates the spelling out by letting us decompose properties like G into $\mathrm{F}$ and non-F parts. Doing so allows us to conclude that an object with property $\mathrm{G}$ will also

\footnotetext{
choice does not really matter for the purposes of inferring something about $\mathrm{B}$ from $\mathrm{E}_{1}$. Choosing a partition* that includes B as a member just makes the point easier to communicate.

${ }^{15}$ The complex proposition $\mathrm{B} a \mathrm{v} \mathrm{C}_{1} a \ldots \mathrm{v} \mathrm{C}_{\mathrm{m}} a$ need not be thoroughly observational, but at least one of its atomic components, i.e. Ba, will be.

${ }^{16}$ I say 'can confirm' instead of 'confirms' to avoid a controversial issue in confirmation theory, i.e. whether or not derived observational statements always have confirmational power. The received view has been that they do always have such power but Larry Laudan and Jarrett Leplin (1991), amongst others, have challenged this view.
} 
possess a property from a finite selection of mutually disjoint properties (partitioned* from G) that includes $\mathrm{F}$. Thus finding an object with property $\mathrm{F}$ can confirm a theory which predicts the existence of objects with property G. To put things in perspective, suppose ' $G$ ' is an unobservable property and ' $\mathrm{F}$ ' an observable one. Theories supplemented with the auxiliary 'All F's are G's' can be confirmed by observational reports of objects possessing property F.

It is worth noting that auxiliaries $A_{3}$ and $A_{4}$ are not merely stipulated but derived from existing auxiliaries, in this case $A_{1}$. We can similarly derive auxiliaries $A_{5},(x)\left(B x \equiv E_{2} x\right)$, and $A_{6},(x)\left(E_{2} x \equiv\left(B x \vee D_{1} x \vee \ldots v D_{k} x\right)\right)$, from $A_{2}$ to allow Priestly's theory to be directly tested by observations. Indeed, with the help of $\mathrm{A}_{5}$ and $\mathrm{A}_{6}$, Priestly's theory can be confirmed by $\mathrm{B} a$. Since $\mathrm{B} a$ can confirm both theories it cannot be used to discriminate between them. This problem is of no concern to us here since we are frying an altogether different fish. The aim was to show that theories plus suitable auxiliaries can be directly tested via observations, i.e. it was not to show that the presence of black powder discriminates between Lavoisier's and Priestly's theories. At any rate, in terms of theory testing we are not worse off than when we started since $E_{1}$ and $E_{2}$ are also unable to discriminate between the two theories. Moreover, the fact that one observation report cannot adjudicate between two theories (plus associated auxiliaries) does not entail that (1) it cannot adjudicate between those theories and others and (2) all observation reports are similarly impotent. ${ }^{17}$ In the historical case at hand, the observation that some substances gained weight during combustion worked in favour of the oxygen theory by disconfirming the phlogiston theory as it was traditionally construed. Spurred on by this observation some phlogiston theorists supplanted the central view that

\footnotetext{
${ }^{17}$ It is still an open question whether for any given theory there is at least one other theory that no observation report can adjudicate between. This takes us into underdetermination territory. As it is customarily understood, the underdetermination thesis presupposes, contra Basu, that derived observation sentences have confirmational power but of course only towards the empirical models of the theory.
} 
phlogiston was weightless with the view that it had negative weight. The manoeuvre did not ultimately save the phlogiston theory, but it certainly illustrates the fact that an observation report can adjudicate between two theories, in our case the oxygen theory vs. the traditionally construed phlogiston theory.

In a sense what I have argued for is unsurprising. An auxiliary of the form 'evidence $\mathrm{x}$ implies observation $\mathrm{y}$ ' or something weaker like 'evidence $\mathrm{x}$ implies a disjunction one of whose disjuncts is an observation y' is implicit in the scientists' thoughts when they employ an inverse conditional, i.e. when they infer from their observations some evidential report. Indeed, on pain of inconsistency, the scientists must have a biconditional or even an identity relation in mind. They take it that one of the manifestations of iron oxide (or iron calx) is black powder, hence they are in effect accepting a statement like 'An object is iron oxide (or iron calx) iff/= it is black powder with certain observable properties or it is a red-brownish solid with certain observable properties or ...'. ${ }^{18}$ This implicit acceptance puts paid to the view that theories do not entail, predict or even potentially explain observation statements. In any case, as I have shown above, the explicit acceptance of the original auxiliaries is sufficient to establish direct contact between theories and observation reports.

A final qualification is in order. It should be obvious that by 'direct contact' I do not mean anything that violates Duhem's thesis that theories can never be tested in isolation. Rather, I mean that theories plus suitable theoretical auxiliaries can entail, predict and potentially explain observation statements or data. In short, the view developed in this paper is perfectly compatible with various forms of holism. ${ }^{19}$

\footnotetext{
${ }^{18}$ Not all of the disjuncts need be observational.

${ }^{19}$ In my view, some form of partial holism is highly plausible.
} 


\section{Conclusion}

It has not been argued here that theories always make direct contact with the observational ground. Instead, it has been argued that cases like the Lavoisier-Priestly controversy, i.e. cases where an auxiliary of the form 'observation $\mathrm{y}$ implies evidence $\mathrm{x}$ ' is already present, do make contact. Whether all theories share this feature is itself a broadly empirical matter. I for one am inclined to think that at least in the majority of cases we can find suitable theoretical auxiliaries which together with their respective theories entail, predict and potentially explain the observational data.

\section{References}

Basu, P. K. (2003) 'Theory-ladenness of Evidence: A Case Study from History of Chemistry', Studies in the History and Philosophy of Science Part A, vol. 34, 351-368.

Bogen, J., and Woodward, J. (1988) 'Saving the phenomena', The Philosophical Review, vol. 97, 303-352.

Laudan, L. and Leplin, J. (1991) 'Empirical Equivalence and Underdetermination', Journal of Philosophy, vol. 88, 449-72. 